\title{
Does Situation-Specificity Affect the Operation of Implementation Intentions?
}

DOI:

10.1016/j.beth.2017.08.003

Document Version

Accepted author manuscript

Link to publication record in Manchester Research Explorer

\section{Citation for published version (APA):}

Epton, T., \& Armitage, C. J. (2017). Does Situation-Specificity Affect the Operation of Implementation Intentions? Behavior therapy. https://doi.org/10.1016/j.beth.2017.08.003

\section{Published in:}

Behavior therapy

\section{Citing this paper}

Please note that where the full-text provided on Manchester Research Explorer is the Author Accepted Manuscript or Proof version this may differ from the final Published version. If citing, it is advised that you check and use the publisher's definitive version.

\section{General rights}

Copyright and moral rights for the publications made accessible in the Research Explorer are retained by the authors and/or other copyright owners and it is a condition of accessing publications that users recognise and abide by the legal requirements associated with these rights.

\section{Takedown policy}

If you believe that this document breaches copyright please refer to the University of Manchester's Takedown Procedures [http://man.ac.uk/04Y6Bo] or contact uml.scholarlycommunications@manchester.ac.uk providing relevant details, so we can investigate your claim.

\section{OPEN ACCESS}




\section{Accepted Manuscript}

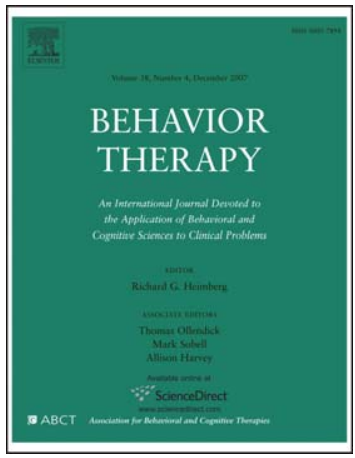

Does Situation-Specificity Affect the Operation of Implementation Intentions?

Tracy Epton, Christopher J. Armitage

PII: $\quad$ S0005-7894(17)30090-4

DOI: $\quad$ doi: $10.1016 /$ j.beth.2017.08.003

Reference: $\quad$ BETH 736

To appear in: $\quad$ Behavior Therapy

Received date: $\quad 7$ October 2016

Accepted date: 2 August 2017

Please cite this article as: Epton, T. \& Armitage, C.J., Does Situation-Specificity Affect the Operation of Implementation Intentions?, Behavior Therapy (2017), doi: $10.1016 /$ j.beth.2017.08.003

This is a PDF file of an unedited manuscript that has been accepted for publication. As a service to our customers we are providing this early version of the manuscript. The manuscript will undergo copyediting, typesetting, and review of the resulting proof before it is published in its final form. Please note that during the production process errors may be discovered which could affect the content, and all legal disclaimers that apply to the journal pertain. 
Does Situation-Specificity Affect the Operation of Implementation Intentions?

\author{
Tracy Epton
}

University of Manchester

Christopher J. Armitage

University of Manchester

Author Note

Correspondence to Tracy Epton PhD., Manchester Centre for Health Psychology, University of Manchester; Christopher J. Armitage PhD., Manchester Centre for Health Psychology, University of Manchester. epton.tracy@gmail.com.

Christopher J. Armitage, Manchester Centre for Health Psychology, University of Manchester; School of Psychological Sciences, University of Manchester, Coupland I, Coupland Street, Oxford Road, Manchester, M13 9PL. 


\begin{abstract}
Interventions that encourage people to link critical situations with appropriate responses (i.e., “implementation intentions") show promise in increasing physical activity.

The study tested whether implementation intentions designed to deal with generic situations are more effective than implementation intentions designed to respond to specific situations.

133 participants either: (a) formed implementation intentions using a volitional help sheet with 10 critical situations (i.e., standard volitional help sheet); (b) formed implementation intentions using a volitional help sheet with one generic situation (i.e., single situation volitional help sheet); or (c) did not form implementation intentions (i.e. control condition).

Participants who formed implementation intentions reported more physical activity and greater self-regulation, than those in the control condition. There were no differences between participants who were provided with one generic critical situation and those who were provided with 10 specific critical situations.

Implementation intentions successfully increased self-reported physical activity irrespective of critical situation specificity. The implication is that implementation intention-based interventions are robust and require minimal tailoring.
\end{abstract}

KEYWORDS: Volitional help sheet; Implementation intentions; Physical Activity; Selfregulation 


\section{Does Situation-Specificity Affect the Operation of Implementation Intentions?}

\section{Physical Inactivity and Health}

Physical inactivity contributes to numerous physical (e.g., ischaemic heart disease, stroke, type 2 diabetes, various cancers) and mental (e.g., psychological distress, depression) health conditions and is responsible for $9 \%$ of premature deaths worldwide (HSE, 2012; Lee et al., 2012). Physical inactivity is also commonplace: In England, $45 \%$ of women and 33\% of men aged 16 and over were not meeting the physical activity guidelines in 2012; moreover, $26 \%$ of women and $19 \%$ of men were classified as inactive (HSE, 2012). There is a need to develop tools that both increase physical activity and are quick and easy to administer in a large population.

Interventions for physical activity typically include multiple behaviour change techniques (BCTs) delivered in multiple sessions by a health care professional. For example, a meta-analysis of physical activity interventions showed that on average 6.6 BCTs were used per intervention, $94 \%$ of interventions consisted of multiple sessions over an average of 24 weeks, and $46 \%$ of interventions were delivered by a health care professional (Michie et al., 2009). Interventions that consist of multiple sessions delivered by a health care professional are time consuming and expensive, and the simultaneous delivery of multiple BCTs obscures any unique effects of individual BCTs. It would therefore be valuable to develop less costly interventions that are based upon the BCTs that have been shown to be most effective.

Concurrent with the growing crisis surrounding physical inactivity, a large-scale nationally-representative survey found that $95 \%$ of UK adults thought physical activity was important for health and 67\% wanted to do more physical activity (HSE, 2007). These figures 
show that many people are motivated to become physically active and may benefit from interventions that focus on translating motivation into action.

\section{Implementation Intentions}

Implementation intentions (Gollwitzer, 1993) are one means of translating motivation into action. When people form implementation intentions (Gollwitzer, 1993), they are asked to link critical situations ("if"s") with appropriate responses ("then's"). The principle is that by linking critical situations (e.g., "if it is lunchtime") with appropriate responses, the response (e.g., "then I will go for a run”) will be triggered automatically when the critical situation (e.g., lunchtime, in the above example) is encountered in the future. Implementation intention-based interventions such as these have increased physical activity in cancer survivors (McGowan et al., 2013), people with myocardial infarctions (Luszczynksa, 2006), people with spinal cord injuries (Latimer et al., 2006), in healthy populations (Andersson \& Moss, 2011, Prestwich et al., 2009), people with low socioeconomic status (Armitage \& Arden, 2010) and among school children (Armitage \& Sprigg, 2010). Recent meta-analyses have indicated that implementation intentions are effective at increasing physical activity. Belanger-Gravel et al. (2013) found nineteen studies, which had an overall small effect at follow-up, $d=.24$, and a slightly larger effect, $d=.31$, when physical activity was measured immediately after the last intervention session. Carraro and Gaudreau (2013) found eight studies that showed a large overall unique effect of implementation intentions, $d=1.03$, on physical activity.

\section{The Volitional Help sheet}

Volitional help sheets (e.g., Armitage, 2008) give participants the raw ingredients with which to form implementation intentions. Volitional help sheets present participants with a choice of specific critical situations and appropriate responses based on Prochaska and 
DiClemente's (1983) transtheoretical model. The situations and responses are presented in two columns, and participants are asked to draw a line between a situation that is relevant to them and a response that they think will work for them, thus linking critical situation with appropriate response. Participants in the control condition are similarly presented with a volitional help sheet but are asked to tick critical situations and appropriate responses that are relevant. This ensures that participants in the control group are exposed to the same situations and responses as the intervention group, but without forming a link between the situation and the response.

Volitional help sheets have been used successfully to improve a range of behaviors: to increase quitting in smokers (Armitage, 2008, 2016), reduce alcohol consumption in the general population (Armitage \& Arden, 2012), reduce binge-drinking in students (Arden \& Armitage, 2012), reduce speeding among drivers (Brewster et al., 2015), increase weight loss (Armitage et al., 2014), reduce suicidal ideation and behavior among patients hospitalized after self-harming (Armitage et al., 2016) and increase physical activity (Armitage \& Arden, 2010).

The physical activity volitional help sheet consists of up to: (a) 20 situations (e.g., If I'm tempted not to be physically active because I am busy) adapted from Marcus et al. (1992a) and Hausenblas et al.'s (2001) temptation not to exercise scales, and (b) 20 appropriate responses adapted from Marcus et al.’s (1992b) processes of change questionnaire. In Armitage and Arden's (2010) study, manual workers were randomized either to form implementation intentions using the volitional help sheet or not. Extent of engaging in moderate physical activity was measured prior to and at one-month after the intervention. Participants in the intervention group reported a significant increase in their moderate physical activity after one-month compared to the control group $(d=.14)$. 


\section{Situation Specificity}

A strength of the volitional help sheet is that the cues and responses are provided for the participant ensuring that the plan has appropriately formed situations and solutions. This is important as health behavior change was found to be more successful when the solutions used in the implementation intentions are specific (de Vet, Oeneman \& Brug, 2011; van Osch, Lechner, Reubsaet \& De Vries, 2010). There has been little research on the specificity of the situations; one study found that people tended to neglect the situation when forming an implementation intention (de Vet, Oeneman \& Brug, 2011).

However, one potential limitation of the standard volitional help sheet (and implementation intentions generally) with it including many situations to choose from that could: (a) create a burden for the participant, and (b) preclude responses being activated when a non-specified situation occurs. Consistent with the latter line of reasoning, there is laboratory evidence to suggest that people are worse at identifying additional cues to action after they have formed an implementation intention. Parks-Stamm et al. (2007) were interested in the speed with which participants were able to identify five-letter words after forming implementation intentions (i.e., a measure of the salience of a critical situation). In one study, participants were asked to form implementation intentions that would enable them to react to particular words (e.g., "if I hear the word 'Laura,' then I will immediately press the letter ' $L$ '"); in another study, participants were asked to form implementation intentions that would react to the number of letters in the word (e.g., "if I hear the word 'Danny,' then I will immediately press the ' 5 '"). Participants in the study in which they were asked to focus on words were faster at identifying the cued words, but were slower to identify other five letter words. In contrast, when participants were asked to react to the number of letters, the effects generalized to other five-letter words (Parks-Stamm et al., 2007). A further study found that, implementation intentions formed in one task still attracted attention in a further unrelated 
task and that participants were unaware of this effect and thus did not disengage from the implementation intention even though it was detrimental (Wieber \& Sassenberg, 2006). From a volitional help sheet perspective, it is possible that selecting a situation such as "If I'm tempted not to be physically active because I am busy" would activate the chosen appropriate response to perform physical activity if the person was feeling too busy, but would not activate an appropriate response if the person was tempted not to exercise because it was cold outside. Concurrently, the person would not realise that their initial plan was interfering so would not take steps address this (e.g., by forming a new implementation intention).

However, the volitional help sheet can be amended to address the problem of implementation intentions narrowing people's attention to other opportunities to act. The volitional help sheet identifies several situations starting with an identical generic stem (e.g., "if I am tempted not to be physically active because..."), which are qualified by more specific affective (e.g., “... I am depressed”), lifestyle (e.g., “... I am busy”), environmental (e.g., "... it is cold outside”), and social (e.g., “... I am because I am spending time with friends or family who do not exercise") situations. Using the generic stem alone would mean that only a single cue is activated thus potentially ameliorating the problem of reduced attention to other situations. The present study compares a control condition with volitional help sheets that have just a single generic situation (i.e., "if I am tempted not to be physically active...") with the standard volitional help sheet that specifies several situations. Thus, the principal aim of the present research is to test the effects of situation specificity on the ability of implementation intentions to increase physical activity.

\section{Potential Mediating Effects}

Laboratory studies have identified increased accessibility to a cue and automatic activation of the appropriate response (Hagger \& Luszczynska, 2014) as the mechanisms 
behind the success of implementation intentions. Although these mechanisms are not yet amenable to direct measurement in the field, there is evidence that the effects of implementation intentions on behavior change can be explained by the effects of increased cue accessibility and cue-response links on self-regulation. Specifically, Armitage (Armitage, 2015) postulated that: (a) self-monitoring is increased by the reallocation of executive resources that are freed up because of the increased automatic activation of the implementation intention, (b) specifying critical situations increases awareness of standards, and (c) the automaticity of an appropriate response boosts self-regulatory effort. The study found that increased self-monitoring mediated the effect of the intervention on behaviour (Armitage, 2015). A secondary aim of the present study is therefore to examine if selfregulation mediates the effect of the two volitional help sheets on physical activity.

\section{Summary}

The present study builds on previous research by testing: (a) the effects of situation specificity on the ability of implementation intentions to boost physical activity, and (b) whether self-regulation mediates the effects of implementation intentions on behavior change. The hypotheses are that: (a) the standard implementation intention condition and the single generic critical situation implementation intention condition will be more effective at increasing physical activity than the control condition, (b) the single generic critical situation condition will be more effective than the standard implementation intention condition (see Parks-Stamm et al., 2007), and (c) improved self-regulation will mediate the effect of implementation intentions on behavior.

\section{Method}

\section{Participants}


Approximately two-hundred people from two office buildings in the North West of England were invited to take part in a study regarding personal and social beliefs about physical activity - all people working in the office blocks were eligible. As a convenience sample was used data collection ended when all invited people were given an opportunity to respond. 133 participants took part in the study; attrition was $1.50 \%$ as two participants failed to complete the physical activity measures (see figure 1 ). The sample was aged $18-63$ years old $(M=36.36, S D=12.50), 57 \%$ were female and $78 \%$ were white.

\section{Procedure}

Potential participants were invited to take part in the study by a supervisor at each of the sites who distributed participant information sheets (the supervisor was blinded to the purpose of the study). The participant information sheet described the purpose of the study was "to understand the physical activity of people in your workplace", and what the study would entail (i.e., "You will be asked to fill in two questionnaires") and included details on consent, right to withdraw ("You do not have to take part in this study. If you decide to take part, and then later change your mind, either before or during the study, you can ask not to take part any longer without explaining why, and if you wish, your questionnaires will be destroyed. After the questionnaires have been collected in, there will be no way of knowing which is yours, so it will not be possible to identify or delete your questionnaire"). The health behavior questionnaires including the different versions of the volitional help sheet (ordered sequentially e.g., a repetition of control, standard volitional help sheet, single-situation volitional help sheet - so every three consecutive people received a different condition) were made available to individual potential participants in the staff room of their respective office buildings. Participants were instructed to take and complete the questionnaires and anonymously place these in a box for the attention of the supervisor. After two months, an identical follow-up health behavior questionnaire was distributed and collected in the same 
manner, and matched up to the initial questionnaire (by use of a self-generated identification code). The data were collected between May and August 2013. No incentives were offered for taking part.

\section{Materials}

All measures were taken at baseline and two months follow-up.

Health behavior questionnaire. The primary outcome was physical activity at twomonth follow-up. Current level of physical activity was measured using the Short Form of the International Physical Activity Questionnaire (IPAQ-SF; Booth, 2000). Respondents were asked to indicate how many times, and for how long, they had engaged in vigorous ("activities that take hard physical effort and make you breathe much harder than normal") and moderate physical activities ("activities that take moderate physical effort and make you breathe somewhat harder than normal"), as well as walking in the past 7 days. Responses were converted into METs (metabolic equivalent of task) to provide a score for each type of physical activity and a total IPAQ score. An additional secondary question asked about time spent being sedentary.

State self-regulation was measured using six items on a 7-point scale (strongly disagree - strongly agree) e.g., "During the last month, I have consistently monitored myself to check that I am being sufficiently physically active" that formed three subscales. (Sniehotta et al., 2005). The subscales were state self-monitoring (baseline Spearman's rho = $.38, p<.01$; follow-up Spearman's rho $=.53, p<.01$ ), state awareness of standards (baseline Spearman's rho $=.44, p<.01$; follow-up Spearman's rho $=.65, p<.01)$, and state effort (baseline Spearman's rho $=.80, p<.01$; follow-up Spearman's rho $=.89, p<.01$ ). Demographic questions regarding age, gender and ethnicity were also included. 
Volitional help sheets. The standard physical activity volitional help sheet comprised two columns with a list of ten situations in column one (e.g., "If I'm tempted not to be physically active when I'm under a lot of stress ...") and a list of ten responses in column two (e.g., "then I will remember how warnings about the health hazards of inactivity move me emotionally"; Armitage \& Arden, 2010). Participants were asked to draw a line to link situations that tempted them not to be physically active to solutions that they thought would work for them.

The single-situation volitional help sheet has an identical layout to the standard volitional help sheet but with just one situation "If I am tempted not to be physically active..." in column one. Participants were asked to link the situation to as many solutions as they thought would work for them.

The control volitional help sheet is identical to the standard volitional help sheet but asked participants to tick situations and solutions that were personally relevant to them. Note that participants in the control condition are not asked to form implementation intentions because they are not asked to link situations with responses (Gollwitzer, 1993).

\section{Analysis}

The data were analysed using SPSS v. 22; as the data was highly skewed and resistant to transformations the Kruskal-Wallis test for independent samples was used to determine differences between conditions (3 levels: control, standard and single-situation) in overall physical activity, vigorous activity, moderate activity, walking, sedentary behaviour and selfregulation (to account for change from baseline to follow-up residuals were calculated by regressing baseline scores onto the follow up scores). Pairwise comparisons were conducted using Mann-Whitney U tests comparing all conditions against one another (a conservative alpha of $p<.017$ was used due to multiple comparisons; the traditional value of .05 was 
amended based on the number of tests conducted). The primary analysis was conducted on all those who completed baseline and follow up questionnaires. A secondary analysis was conducted using a per protocol analysis whereby only those participants who had completed one of the interventions were compared to the control group.

The Hayes and Preacher (2013) mediation technique for multi-categorical variables (each of the experimental volitional help sheet conditions will be compared to a reference category, i.e., the control condition) was planned to be used to determine if self-regulation is a mediator of the relationship between the volitional help sheets and physical activity; however, as the data was non-normally distributed these analyses were not conducted.

The study used a convenience sample so recruitment stopped when all workers at the two offices had been approached. The sample size achieved was large enough to detect the medium to large effects that are typically found using this type of manipulation ${ }^{1}$.

\section{Ethical Approval}

This study was reviewed and granted ethical approval by University of Manchester Senate Committee on the Ethics of Research on Human Beings (Reference: ethics/12352 amendment).

\section{Results}

\section{Allocation Check}

The three conditions did not differ on gender, $\chi^{2}(1, N=106)=4.24, p=.120$, ethnicity, $\chi^{2}(1, N=125)=2.14, p=.343$, or age, $F(2,124)<1$. The conditions did not differ on baseline measures of physical activity, $H(2, N=131)=5.13, p=.077$, [walking, $H(2, N=$ $131)=1.57, p=.455$, moderate activity, $H(2, N=131)=2.54, p=.282$, vigorous activity, 
$H(2, N=131)=3.19, p=.202]$ or self-regulation, $H(2,131)=4.19, p=.123$ (See Table 1). However, the conditions differed in sedentary behavior, $H(2, N=123)=6.13, p=.047$.

\section{Effects of Implementation Intention Formation on Physical Activity}

The conditions differed in total physical activity at follow-up, $H(2, N=131)=10.48$, $p=.005$ (see Table 2 for descriptive statistics). Exploration of the effects showed that the control condition differed significantly from the standard volitional help sheet (MannWhitney $U=641.50, p=.005)$ and the single-situation volitional help sheet (Mann-Whitney $U=658.00, p=.007)$ in support of the first hypothesis. Contrary to the second hypothesis the two interventions did not differ from each other (Mann-Whitney $U=870.50, p=.918$ ).

Decomposition of these effects revealed that the differences in overall physical activity were attributable to increases in vigorous physical activity, $H(2, N=131)=12.83, p=.002$, rather than walking, $H(2, N=113)=.25, p=.881$, or moderate physical activity, $H(2, N=124)=$ $3.55, p=.169$. Participants in the standard and the single-situation volitional help sheet conditions reported significantly more vigorous physical activity (Mann-Whitney $U=534.50$, $p=.001 ;$ Mann-Whitney $U=612.5, p=.005$, respectively) than those in the control condition. The two experimental conditions did not differ significantly from one another in terms of follow-up physical activity (Mann-Whitney $U=741.00 ; p=.694$ ). Sedentary behavior did not differ significantly, $H(2, N=112)=.68, p=.710$, between the conditions. The secondary per protocol analysis found similar results (see Table 3).

\section{Effects of Implementation Intention Formation on Self-Regulation}

In partial support of hypothesis three, there were significant differences between conditions in self-regulation at follow-up for the overall scale, $H(2, N=131)=15.77, p<$ .001 , awareness of standards, $H(2, N=132)=15.55, p<.001$, and effort, $H(2, N=132)=$ $15.17, p<.001$, but not for self-monitoring, $H(2, N=132)=5.37, p=.068$. For both 
awareness of standards and effort participants in the standard condition (Mann-Whitney $U=$ 538.00, $p<.001 ;$ Mann-Whitney $U=524.50, p<.001)$ and the single situation condition (Mann-Whitney $U=651.50, p=.004 ;$ Mann-Whitney $U=714.50, p=.017$ ) reported greater self regulation than those in the control condition. There were no differences in awareness of standards between the standard and single situation condition for either awareness of standards (Mann-Whitney $U=794.50, p=.340$ ) or effort (Mann-Whitney $U=725.00, p$ $=.118$ ). A mediation analysis could not be conducted as the data were non-normally distributed.

\section{Discussion}

\section{Summary}

The present study tested whether the specificity with which a situation is stated affects the operation of implementation intentions. The key finding was that specifying a generic single-situation was just as effective as being given the opportunity to choose from 10 specific situations in increasing vigorous physical activity, over a two-month follow-up, in comparison to the control condition. The volitional help sheet increased weekly vigorous physical activity by 239.25 METs (30 minutes) in the standard condition, 707.15 METs (88 minutes) in the single-situation condition in comparison to a decrease of 540.98 (68 minutes) in the control condition. The following discussion considers the implications both for public health and theory.

\section{Effects on Physical Activity}

The relationship between physical activity and health is dose dependent meaning that even modest increases in physical activity lead to corresponding improvements in health. This includes risk reduction for coronary heart disease, cardiovascular disease, stroke, 
hypertension, diabetes, and metabolic syndrome (WHO, 2010). Increases in vigorous physical activity, such as those as a result of the physical activity volitional help sheet have an impact on long term health due to the improved health benefits of vigorous over moderate physical activity (Bull \& the expert working group, 2010).

The effectiveness of the two versions of the physical activity volitional help sheet contribute to an emerging body of evidence that the volitional help sheet is a useful means of bringing about behavior change (Arden \& Armitage, 2012; Armitage, 2015; Armitage, 2008; Armitage et al., 2014; Armitage et al., 2016; Armitage \& Arden, 2012; Armitage \& Arden, 2010; Brewster et al, 2015). This study also builds on previous research by Armitage and Arden (2010) who found that the volitional help sheet was effective in increasing moderate physical activity in manual workers over one month. The present study found that the volitional help sheet was effective in increasing physical activity in office workers up to two months after the intervention.

The physical activity volitional help sheet is a tool that can be recommended by practitioners to their patients to encourage increases in physical activity. The volitional help sheet is low cost, quick and easy to deliver and allows the user to tailor their plans to address relevant situations and to choose solutions that work for them. Additionally, the singlesituation volitional help sheet reduces the burden on the user, compared to the standard volitional help sheet.

\section{Theoretical Implications}

The single-situation volitional help sheet is no more effective than the standard volitional help sheet, which suggests that the multiple implementation intentions are also effective at allowing recognition of sufficient cues to action. This reflects the results of a recent study that suggests that plans that specify a particular situation are only a hindrance in 
recognising other opportunities to act on a goal when there are constraints such as time (Masicampo \& Baumeister, 2012) and a study that found overall specificity of the entire plan was related to increased behaviour change (de Vet et al., 2011). However, the parsimony of the single-situation volitional help sheet is a potential added strength.

\section{Limitations}

The present findings support the effectiveness of a single-situation volitional help sheet; however, it is important to consider the limitations of the study. First, the dependent measures used, although reliable and validated, were self-report. Self-report measures are susceptible to social desirability effects - however, because of our allocation procedure, any social desirability effects should be equivalent between groups. Second, although the length of the follow-up in the present study was twice as long as previous studies, according to theory, two-months is insufficient time to show maintenance of behavior (e.g., Prochaska \& DiClemente, 1983) and it would be valuable to show that the effects were sustained in the longer term. Third, the study used sequential allocation to conditions rather than randomization so causation cannot be inferred. Fourth, a no-treatment control was not used therefore it is not possible to determine if the effects on physical activity are due to increases in the intervention condition or decreases in the control condition. There are two lines of evidence that the effect shown in this study is genuine: (a) previous studies that have compared standard volitional help sheets, active control and no-treatment have found null effects between the no-treatment and the active control (Armitage, 2008; Arden \& Armitage, 2012) and (b) further studies have found no detrimental effects of the active control condition across a range of behaviors (Arden \& Armitage, 2010; Arden \& Armitage, 2012; Armitage, 2008; Armitage, 2015; Armitage \& Arden 2012; Armitage et al., 2014). 
A fifth possible limitation is that there seemed to be a high rate of non-compliance with the intervention as $26.74 \%$ of those allocated to the intervention conditions did not fill in the volitional help sheets as instructed. However, it is possible that those participants did use an alternative method to drawing a line (e.g., linking the completed sentence by reading it to themselves) to link the situations and solutions in the manner required for successful implementation intentions to be made. As the per protocol analysis showed the same results as the main analysis this suggests that those participants did indeed use another method to consolidate the implementation intention. Indeed, there is evidence emerging that merely reading an implementation intention is merely sufficient to change people's behaviour (Armitage \& Arden, 2016).

\section{Conclusion}

In conclusion, the single-situation volitional help sheet is as effective in increasing physical activity as the standard volitional help sheet. The strength of the single-situation version is that the burden to participants is reduced. 


\section{Compliance with Ethical Standards}

\section{Ethical Approval}

All procedures performed in studies involving human participants were in accordance with the ethical standards of the institutional and/or national research committee and with the 1964 Helsinki declaration and its later amendments or comparable ethical standards.

\section{Informed Consent}

Informed consent was obtained from all individual participants included in the study. 


\section{References}

Andersson E. K., \& Moss, T. P. (2011). Imagery and implementation intention: A randomized controlled trial of interventions to increase exercise behavior. Psychology of Sport \& Exercise, 12, 63-70. doi:10.1016/j.psychsport.2010.07.004.

Arden, M. A., \& Armitage, C. J. (2012). A volitional help sheet to reduce binge drinking in students: A randomized exploratory trial. Alcohol and Alcoholism, 47, 156-159. doi: 10.1093/alcalc/agr164.

Armitage, C.J. (2008). A volitional help sheet to encourage smoking cessation: A randomized exploratory trial. Health Psychology, 27, 557-566. doi: 10.1037/0278-6133.27.5.557.

Armitage, C. J. (2015). Field experiment of a very brief worksite intervention to improve nutrition among health care workers. Journal of Behavioral Medicine.

Armitage, C. J. (2016). Evidence that implementation intentions can overcome the effects of smoking habits. Health Psychology, 35, 935-943. doi: 10.1037/hea0000344

Armitage, C. J., Abdul Rahim, W., Rowe, R., \& O’Connor, R. C. (2016). An exploratory randomized trial of a simple, brief psychological intervention to reduce subsequent suicidal ideation and behaviour in patients hospitalized for self-harm. British Journal of Psychiatry, 208, 470-476.

Armitage, C. J., \& Arden, M. A. (2010). A volitional help sheet to increase physical activity in people with low socioeconomic status: A randomized exploratory trial. Psychology and Health, 25, 1129-1145. doi: 10.1080/08870440903121638

Armitage, C. J., \& Arden, M. A. (2012). A volitional help sheet to reduce alcohol consumption in the general population: A field experiment. Prevention Science, 13, 635-643. doi: 10.1007/s11121-012-0291-4. 
Armitage, C. J., \& Arden, M. A. (2016). Enhancing the effectiveness of alcohol warning labels with a self-affirming implementation intention. Health Psychology, 35, 11591163. doi: 10.1037/hea0000376.

Armitage, C. J., Norman, P., Noor, M., Alganem, S., \& Arden, M. A. (2014). Evidence that a very brief psychological intervention boosts weight loss in a weight loss program. Behavior Therapy, 45, 700-707. doi: 10.1016/j.beth.2014.04.001.

Armitage, C. J., \& Sprigg, C. A. (2010). The roles of behavioural and implementation intentions in changing physical activity in young children with low socioeconomic status. Journal of Sport \& Exercise Psychology, 32, 359-376.

Armitage, C. J., Wright, C. L., Parfitt, G., Pegington, M., Donnelly, L. S., \& Harvie, M. N. (2014). Self-efficacy for temptations is a better predictor of weight loss than motivation and global self-efficacy: Evidence from two prospective studies among overweight / obese women at high risk of breast cancer. Patient Education and Counselling, 95, 254-258. doi: 10.1016/j.pec.2014.01.015.

Belanger-Gravel, A., Godin, G., \& Amireault, S. (2013). A meta-analytic review of the effect of implementation intentions on physical activity. Health Psychology Review, 7, 23 54.

Booth, M. L. (2000). Assessment of physical activity: An international perspective. Research Quarterly of Exercise and Sport, 71, s114-120.

Brewster, S. E., Elliott, M. A., \& Kelly, S. W. (2015). Evidence that implementation intentions reduce drivers' speeding behavior: Testing a new intervention to change driver behavior. Accident Analysis and Prevention, 74, 229-242. doi: 10.1016/j.aap.2014.11.006 
Bull, F. C. \& Expert Working Groups (2010). Physical activity guidelines in the UK: Review and recommendations; Appendices. Retrieved from https://www.gov.uk/government/uploads/system/.../dh_128257.pdf.

Carraro, N. \& Gaudreau., P. (2013). Spontaneous and experimentally induced action and coping planning for physical activity. Psychology of Sport and Exercise, 14, 228-248.

de Vet, E., Oeneman, A., \& Brug, J. (2011). More or better: Do the number and specificity of implementation intentions matter in increasing physical activity? Psychology of Sport \& Exercise, 12, 471-477.

Gollwitzer, P. M. (1993). Goal achievement: The role of intentions. European Review of Social Psychology, 4, 141-185.

Hagger, M. S., \& Luszczynska, A. (2014). Implementation intention and action planning interventions in health contexts: State of the research and proposals for the way forward. Applied Psychology: Health \& Well-Being, 6, 1-14. doi: 10.1111/aphw.12017.

Hausenblas, H. A., Nigg, C. R., Dannecker, E. A., Symons Downs, D., Ellis Gardner, R., Fallon, E. A., ... Loving, M. G. (2001). A missing piece of the transtheoretical model applied to exercise: Development and validation of the temptation to not exercise scale. Psychology and Health, 16, 381-390. doi: 10.1080/08870440108405514.

Hayes, A. F., \& Preacher, K. J. (2014). Statistical mediation analysis with a multicategorical independent variable. British Journal of Mathematical and Statistical Psychology, 67, 451-470. doi: 10.1111/bmsp.12028.

HSE (2012). Physical activity in adults. Retrieved from http://www.hscic.gov.uk/catalogue/PUB13218. 
HSE (2007). Healthy lifestyles, attitudes and behavior. Retrieved from http://www.hscic.gov.uk/pubs/hse07healthylifestyles.

Latimer, A. E., Martin Ginis, K. A., \& Abour, K. P., (2006). The efficacy of an implementation intention intervention for promoting physical activity among individuals with spinal cord injury: A randomized controlled trial. Rehabilitation Psychology, 51, 273-280. doi: 10.1037/0090-5550.51.4.273

Lee, I. M., Shiroma, E. J., Lobelo, F., Puska, P., Blair, S. N., \& Katzmarkzyk, P. T. (2012). Effect of physical inactivity on major non-communicable diseases worldwide: an analysis of burden of disease and life expectancy. Lancet, 380, 219-29. doi:10.1016/S01406736(12)61031-9.

Luszczynska, A. (2006). An implementation intentions intervention, the use of a planning strategy, and physical activity after myocardial infarction. Social Science and Medicine, 62, 900-908. doi: 10.1016/j.socscimed.2005.06.043.

Marcus, B. H., Rossi, J. S., Selby, V. C., Niaura, R. S., \& Abrams, D. B. (1992a). The stages and processes of exercise adoption and maintenance in a worksite sample. Health Psychology, 11, 386-395.

Marcus, B. H., Selby, V. C., Niaura, R. S., \& Rossi, J. S. (1992b). Self-efficacy and the stages of exercise behavior change. Research Quarterly for Exercise and Sport, 63, 60-66.

Masicampo, E. J., \& Baumeister, R. F. (2012). Committed but closed-minded: When making a specific plan for a goal hinders success. Social Cognition, 30, 37-55. doi: $10.1521 /$ soco.2012.30.1.37 
McGowan, E. L., North, S., \& Courneya, K. S. (2013). Randomized controlled trial of a behavior change intervention to increase physical activity and quality of life in prostate cancer survivors. Annals of Behavioral Medicine, 46, 382-393. doi: $10.1007 / \mathrm{s} 12160-013-9519-1$

Michie, S., Abraham, C., Whittington, C., \& McAteer, J. (2009). Effective techniques in healthy eating and physical activity interventions: A meta regression. Health Psychology, 28, 690-701. doi: 10.1037/a0016136.

Park-Stamm, E. J., Gollwitzer, P. M., \& Oettingen, G. (2007). Action control by implementation intentions: Effective cue detection and efficient response initiation. Social Cognition, 25, 248-266.

Prestwich, A. J., Perugini, M., \& Hurling, R. (2009). Can the effects of implementation intentions on exercise be enhanced using text messages? Psychology \& Health, 24, 677-687. doi: 10.1080/08870440802040715.

Prochaska, J.O., \& DiClemente, C.C. (1983). Stages and processes of self-change in smoking: Toward an integrative model of change. Journal of Consulting and Clinical Psychology, 51, 390-395.

Sniehotta, F. F., Scholz, U., \& Schwarzer, R. (2005). Bridging the intention-behavior gap: Planning, self-efficacy, and action control in the adoption and maintenance of physical activity. Psychology \& Health, 20, 143-160. doi: 10.1080/08870440512331317670.

van Osch, L., Lechner, L., Reubsaet, A., \& de Vries, H. (2010). From theory to practice: An exploratory study into the instrumentality and specificity of implementation intentions. Psychology \& Health, 25, 351-364. 
WHO (2010). Global recommendations on physical activity for health. Retrieved from: http://www.who.int/dietphysicalactivity/publications/pa/en/.

Wieber, F., \& Sassenberg, K. (2006). I can't take my eyes off of it - attention attraction effects of implementation intentions. Social Cognition, 24, 723-752. 


\section{Endnotes}

${ }^{1}$ The initial statistical power was based on a calculation using $\mathrm{G}^{*}$ Power for ANCOVA with 3 groups at .80 power for a medium effect the sample size would be $N=158$ and for a large effect $N=64$; however, the amount of data collected was limited by the response rate of the two organizations. 


\section{Tables}

Table 1 Baseline characteristics of the sample

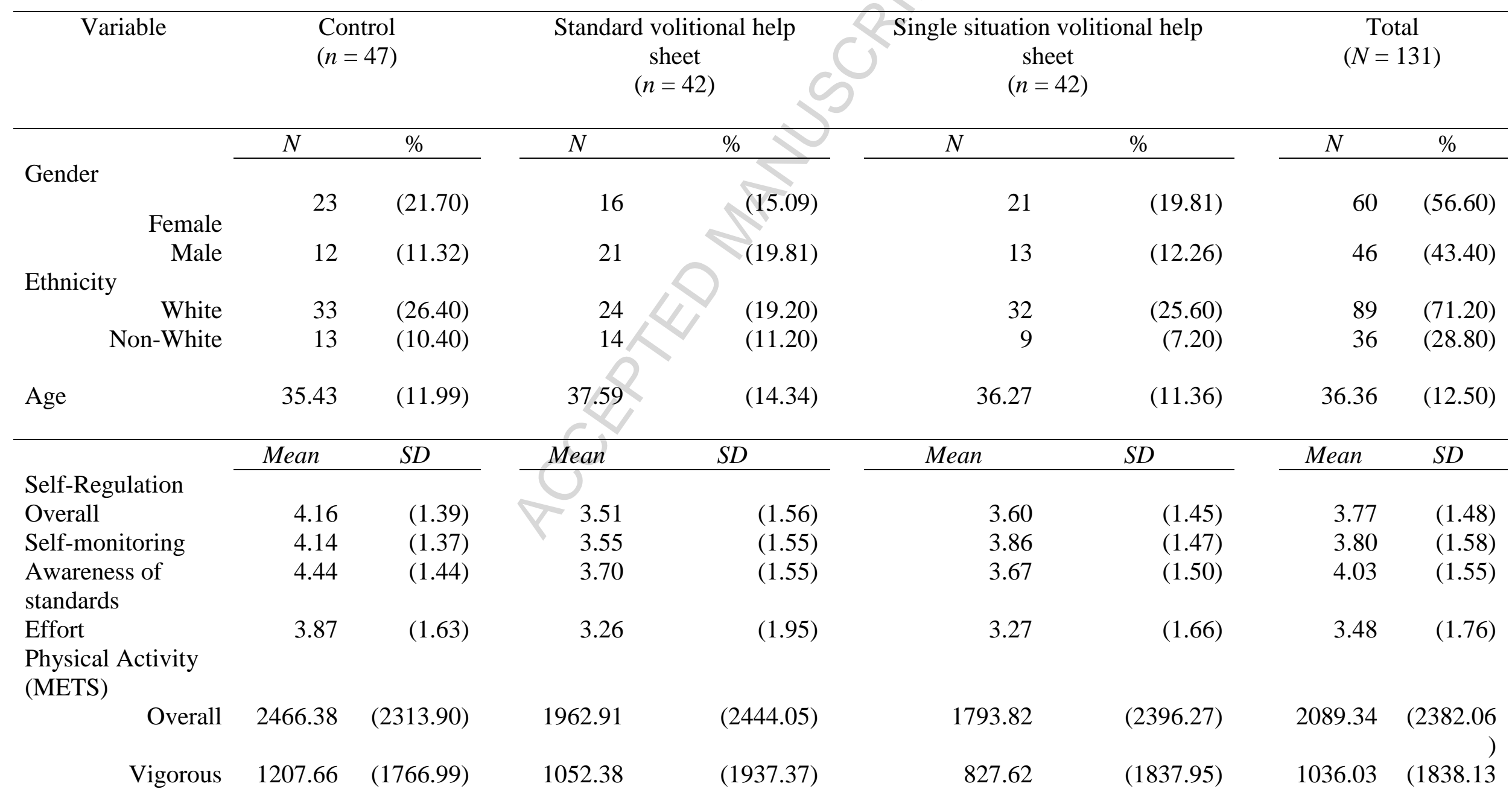




\begin{tabular}{|c|c|c|c|c|c|c|c|c|}
\hline Moderate & 556.60 & $(985.51)$ & 311.43 & $(926.60)$ & 503.81 & (897.07) & 461.07 & $(937.75)$ \\
\hline $\begin{array}{l}\text { Sedentary } \\
\text { Behavior }\end{array}$ & 469.05 & (184.86) & 492.14 & (194.65) & 391.15 & $(205.21)$ & 452.24 & (197.92) \\
\hline
\end{tabular}

Table 2 Means (standard deviations in parenthesis), $p$ values and self-regulation at follow-up

\begin{tabular}{|c|c|c|c|c|c|c|c|}
\hline Self-Regulation & \multicolumn{2}{|c|}{$\begin{array}{l}\text { Control } \\
(n=47)\end{array}$} & \multicolumn{2}{|c|}{$\begin{array}{l}\text { Standard volitional help sheet } \\
\qquad(n=42)\end{array}$} & \multicolumn{2}{|c|}{$\begin{array}{l}\text { Single situation volitional help } \\
\text { sheet } \\
(n=42)\end{array}$} & $p$ value \\
\hline Overall & 300 & $(15)$ & 453 & $(170)$ & 420 & (17) & $<001$ \\
\hline Self-monitoring & 4.23 & $(1.55)$ & 4.44 & (1.65) & 4.39 & $(1.70)$ & .073 \\
\hline $\begin{array}{l}\text { Awareness of } \\
\text { standards }\end{array}$ & 4.07 & (1.44) & 4.61 & (1.77) & 4.36 & (1.71) & $<.001$ \\
\hline Overall & 1967.93 & (1946.66) & 2468.61 & $(3454.82)$ & 2215.89 & $(2776.80)$ & .005 \\
\hline Vigorous & 910.81 & (1532.56) & 1177.14 & (1940.08) & 1361.90 & $(2514.61)$ & .002 \\
\hline Moderate & 413.62 & $(763.95)$ & 627.14 & (1321.17) & 423.81 & $(731.07)$ & .134 \\
\hline Walking & 643.50 & $(802.95)$ & 664.32 & $(963.20)$ & 430.18 & $(529.70)$ & .704 \\
\hline $\begin{array}{l}\text { Sedentary } \\
\text { Behavior }\end{array}$ & 472.75 & (219.18) & 516.50 & $(205.65)$ & 451.32 & $(222.66)$ & .710 \\
\hline
\end{tabular}


Table 3 Means (standard deviations in parenthesis), $p$ values and self-regulation at follow-up - per protocol analysis

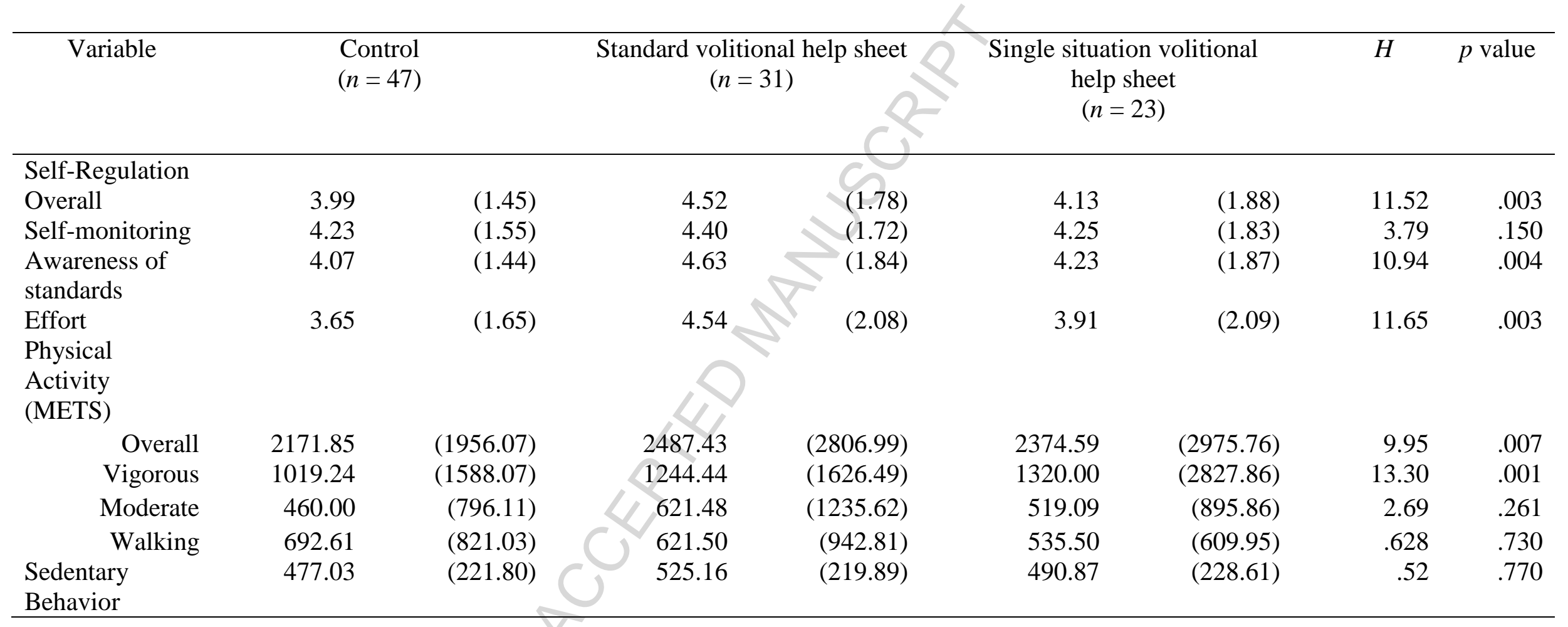




\section{Figure}

Figure 1: Flow of participants through the study

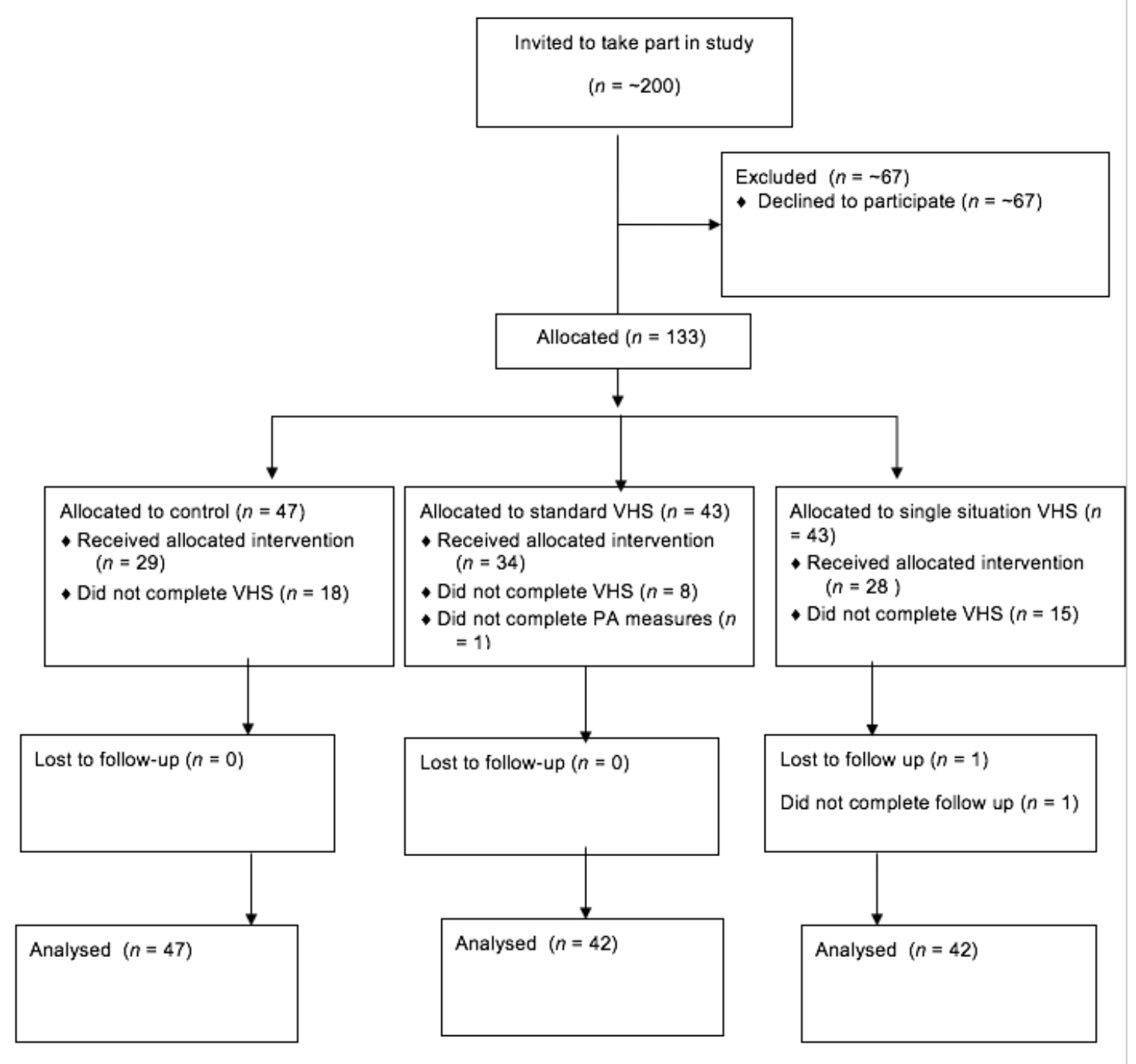


Highlights

- Increased physical activity has a positive health benefit

- Two interventions were compared against a control in increasing physical activity

- The interventions increased vigorous physical activity in particular

- The brief intervention was as effective as the standard intervention 